REVISTA DE CIENCIAS FORENSES DE HONDURAS
Revista de Ciencias Forenses de Honduras ISSN: 2412-8058

ISSN: 2413-1067

revistacienciasforenseshnd@gmail.com

Dirección de Medicina Forense de Honduras Honduras

Rodríguez Maldonado, VJ; Fernández, L; Casanova, A; Alonzo Matamoros, IA Comparación de tres métodos odontológicos para estimación de edad dental en niños hondureños.

Revista de Ciencias Forenses de Honduras, vol. 4, núm. 1, 2018, Enero-Junio, pp. 2-8

Dirección de Medicina Forense de Honduras Honduras 


\title{
Comparación de tres métodos odontológicos para estimación de edad dental en niños hondureños.
}

\section{Comparison of Demirjian, Moorrees and Nolla forensic methods to estimate the dental age of children.}

\author{
Rodríguez Maldonado VJ ${ }^{1 *}$, Fernández L. ${ }^{2}$, Casanova A. ${ }^{3}$ Alonzo-Matamoros IA ${ }^{4}$.
}

\begin{abstract}
${ }^{1}$ Cirujano Dental, Clínica Sonrie, Smile Design Center, Tegucigalpa, Honduras.
${ }^{2}$ Especialista en Prostodoncia e Implantología, Universidad Católica de Honduras, Nuestra Señora Reina de la Paz, Tegucigalpa, Honduras.

${ }^{3}$ Cirujano Dentista y Perito Forense, Dirección de Medicina Forense, Tegucigalpa y Universidad Católica de Honduras, Nuestra Señora Reina de la Paz, Tegucigalpa, Honduras.

${ }^{4}$ Licenciado en Matemáticas, Departamento de Matemáticas, Escuela de Ciencias, Universidad Nacional Autónoma de Honduras.
\end{abstract}

*Correspondencia a Víctor Rodríguez-Maldonado: victor13rodriguez@gmail.com

\section{REFERENCIA}

Rodríguez Maldonado VJ, Fernández L., Casanova A, Alonzo-Matamoros IA. Comparación de tres métodos odontológicos para estimación de edad dental en niños hondureños. Rev. cienc. forenses Honduras. 2018; 4(1):2-8.

Los autores declaran que no tienen conflicto de intereses en la publicación de este artículo.

RECIBIDO: Noviembre 2017

ACEPTADO: Agosto 2018

\section{RESUMEN:}

La estimación de la edad dental reviste de vital importancia en el ámbito forense por sus amplias aplicaciones en los procesos de identificación y su aplicación para estimar la edad cronológica especialmente en personas vivas, en las que no se dispone de pruebas válidas de su fecha de nacimiento. Se compararon los métodos de Dermirjian, Moorees y Nolla para estimación de la edad dental con fines de aplicación forense, para lo cual se seleccionaron 114 radiografías panorámicas de pacientes hondureños por nacimiento, de ambos sexos (57 femeninos y 57 masculinos), con edades cronológicas entre 5 y 17 años que acudieron al Servicio de Ortodoncia del Hospital Odontológico Monseñor Agustín Hombach de la Universidad Católica de Honduras en Tegucigalpa; los pacientes no mostraron agenesias dentales en la hemiarcada inferior izquierda, ni alteraciones en el desarrollo dental; sin tratamientos de apicoformación y sin extracción prematura de primeras o terceras molares, los estadios de maduración se asignaron de acuerdo a la metodología propuesta por Demirjian, Moorrees y Nolla. La edad cronológica se obtuvo de los expedientes, se determinó la exactitud de cada método, calculando la diferencia de las medias entre la edad cronológica y la edad dental estimada por los tres métodos 
mediante una prueba de Wilcoxon, debido a que la muestra no presentó una distribución normal. Morrees $(0.31, D E=1.14)$ y Nolla $(0.1, D E=1.23)$ sobreestiman la edad en el sexo femenino y la subestiman en los masculinos (0.3/-0.2, DE = 1.32/1.35), mientras que el método de Demirjian subestima la edad en ambos sexos (-0.4/-0.4, DE $=1.23 / 1.31$ ). Se evidenció que en la muestra estudiada no hay diferencias estadísticamente significativas entre la edad cronológica y la edad dental estimada por los métodos de Demirjian, Moorees y Nolla.

\section{PALABRAS CLAVE:}

Edad dental; Demirjian; Nolla; Moorrees; edad cronológica, Niños hondureños, odontología forense.

\section{ABSTRACT}

The estimation of the dental age is of vital importance in the forensic field for its vast applications in the identification processes and mostly for the estimation of the chronological age especially in living people, in which there is no proof of their date of birth. To estimate the dental age for forensic application a comparison between the methods of Demirjian, Moorrees and Nolla was held, for which 114 panoramic radiographs of Honduran patients were selected, both genders (57 female and 57 male), with chronological ages between 5 and 17 years old that attended the Orthodontics Department of the Odontological Hospital of the Catholic University of Honduras in Tegucigalpa "Monseñor Agustín Hombach". The inclusion criteria: patients who don't have any dental agenesia in the left inferior hemiarcade, nor alterations in dental development; no apicoformation treatments and no premature extraction of the first or third molars, the maturation stages were assigned by the methodology proposed by Demirjian, Moorrees and Nolla. The chronological age was obtained from the clinical files. The difference between the means of dental age estimated by the three methods and the chronological dental age was calculated by means of a Wilcoxon test, due that the sample didn't have a normal distribution. Morrees $(0.31, s d=1.14)$ and Nolla $(0.1, s d=1.23))$ overestimate the age in the female sex and underestimate it in the male (0.3/$0.2, s d=1.32 / 1.35)$, while the Demirjian method underestimates the age in both sexes $(-0.4 /-0.4$, $s d=1.23 / 1.31)$. It was evidenced that in the sample studied there are no statistically significant differences between chronological age and dental age estimated by the methods of Demirjian, Moorrees and Nolla.

\section{KEYWORDS}

Age estimation; Demirjian; Nolla; Moorrees; Chronological age, Hondurans children; Forensics odontology.

\section{INTRODUCCIÓN}

La estimación de edad dental en Odontología Forense es una pericia de aplicación cotidiana no solo en la identificación de cadáveres o sus restos, sino también es aplicable para estimar la edad cronológica especialmente en personas vivas, como los menores, en los que no se dispone de pruebas válidas de su fecha de nacimiento ${ }^{1}$. Determinar la edad cronológica, reviste de especial importancia, especialmente en menores, por sus implicaciones como referente social, legal y penal ${ }^{2}$. Los métodos de estimación de la edad dental, describen, mediante radiografías panorámicas, el desarrollo morfo dental; el grado de desarrollo, maduración y erupción dental para determinar la edad biológica y hacer una aproximación a la edad cronológica ${ }^{3}$. Entre los métodos más comúnmente utilizados para estimar la edad dental tenemos el de Demirjian ${ }^{4,5}$, el de Willems, que es una adaptación del de Demirjian ${ }^{6}$, el de Nolla ${ }^{7}$ y el de Moorrees ${ }^{8}$. Estos se basan en el hecho de que el desarrollo de los dientes se produce de manera relativamente constante a lo largo de un periodo de tiempo que se extiende desde la etapa fetal hasta aproximadamente los 20 años de edad y se considera que la morfogénesis dental puede 
variar por factores genéticos, como el patrón étnico, el sexo, y por factores epigenéticos, como la distribución geográfica y el medio ambiente ${ }^{1}$. En nuestro país los métodos que más utilizan los peritos forenses para la estimación de edad son los métodos de Demirjian, Moorrees y Nolla por lo que es necesario evaluar si hay diferencias estadísticamente significativas entre la edad cronológica y la edad dental calculada a partir de estos métodos cuando se aplican a poblaciones con características genéticas, nutricionales, socioeconómicas y ambientales como la nuestra.

\section{MÉTODOS}

Se realizó un estudio retrospectivo de 114 pacientes hondureños por nacimiento, entre cinco y 17 años de edad, que acudieron al Servicio de Ortodoncia del Hospital Odontológico Monseñor Agustín Hombach de la Universidad Católica de Honduras en Tegucigalpa; pacientes sanos, sin agenesias dentales en la hemiarcada inferior izquierda, sin alteraciones en el desarrollo dental; sin tratamientos de apicoformación y sin extracción prematura de primeras o terceras molares, que tenían su respectiva radiografía panorámica en el expediente de evaluación odontológica, 57 pertenecían a pacientes del sexo femenino y 57 al sexo masculino. Para estimar la edad dental a la muestra de radiografías seleccionadas se les aplicó el método de Demirjian ${ }^{5,6}$, Moorrees ${ }^{7}$ y Nolla ${ }^{8}$; de acuerdo a la metodología establecida por cada autor; la lectura radiográfica para el estimado de la edad dental fue realizada por un solo observador. La edad cronológica de los pacientes se obtuvo de la fecha de nacimiento consignada en el expediente. Se determinó la exactitud entre la edad dental y la edad cronológica para cada método y se diferenció además por sexo. La exactitud para fines de este estudio se definió como la diferencia entre la edad cronológica y la edad dental; para establecer la significancia de las diferencias entre la edad cronológica y la edad dental estimada por método, se utilizó la prueba de Willcoxon ${ }^{9}$ en virtud que la muestra no presento una distribución normal; la distribución de normalidad de la muestra se calculó con una prueba de Shapiro-Wilk ${ }^{10}$. Se calculó el error estimado por método y sexo, el error estimado es la diferencia entre la estimación del método y el valor real, el método cuyo error se acerca más a cero es el más exacto. A la mediana de la edad cronológica se le resto el valor de la mediana de la edad dental, interpretándose un resultado positivo como una sobrestimación de la edad y un valor negativo como una subestimación de la edad ${ }^{11}$.

\section{RESULTADOS}

La distribución de normalidad de la muestra se determinó mediante la prueba de Shapiro-Wilk la cual indica que esta, no presenta una distribución normal, valor $p=<0,05$. El Cuadro 1, muestra un comparativo entre la edad dental calculada por cada uno de los métodos en relación a la edad cronológica.

Cuadro 1: Cuadro comparativo entre la edad cronológica y la edad dental por método

\begin{tabular}{l|c|c|c|c|c|c}
\hline \multicolumn{1}{c|}{ Método } & \multicolumn{2}{|c|}{ Media } & \multicolumn{2}{c|}{ Mediana } & \multicolumn{2}{c}{ DE } \\
\hline \multicolumn{1}{c|}{$n=114$} & EC & ED & EC & ED & EC & ED \\
\hline Demirjians & $\mathbf{1 2 . 4 3}$ & 12.88 & $\mathbf{1 2 . 6}$ & 13.50 & $\mathbf{2 . 5 9}$ & 2.55 \\
\hline Moorrees & & 11.93 & & 12.50 & & 2.57 \\
\hline Nolla & & 12.49 & & 13.00 & & 2.49 \\
\hline
\end{tabular}

$E \bar{C}=$ Edad cronológica, $E D=$ Edad dental, $\mathbf{D E}=$ Desviación Estándar

El Cuadro 2 muestra la exactitud entre la edad cronológica y la edad dental estimada por método, la cual se calculó utilizando la prueba de Willcoxon; bajo la hipótesis que no hay diferencia significativa entre la edad cronológica y la edad dental, para un valor $p$ menor o igual a 0,05. EI Cuadro 3 muestra la exactitud entre la edad cronológica y la edad dental estimada por método y diferenciada por sexo. El Gráfico 1 compara los errores estimados por método y el Cuadro 4 muestra el error estimado por método y sexo. 
Cuadro 2: Exactitud entre la edad dental y cronológica por método, calculada por la prueba de Willcoxon

\begin{tabular}{lcc}
\hline \multicolumn{1}{c}{ Método } & Estadístico W & P valor $(P=<0,05)$ \\
\hline Demirjian & $7,266.0$ & 0.1231110 \\
Moorrees & $5,905.0$ & 0.2340580 \\
Nolla & $6,610.5$ & 0.8219851 \\
\hline
\end{tabular}

Cuadro $\mathbf{N}^{\circ}$ 3: Exactitud entre la edad cronológica y la estimación de edad dental por método aplicado y diferenciada por sexo.

\begin{tabular}{l|c|ccc|ccc|c}
\hline \multirow{3}{*}{ Método } & \multirow{3}{*}{ Género } & \multicolumn{3}{|c|}{ Edad Cronológica } & \multicolumn{3}{c|}{ Edad Dental } & \\
\cline { 3 - 9 } & & $\boldsymbol{n}$ & Mediana & DE & $\boldsymbol{n}$ & Mediana & DE & $\begin{array}{c}\text { Diferencia de } \\
\text { Medianas (EC-ED) }\end{array}$ \\
\hline \multirow{3}{*}{ Demirjiann } & Femenino & 57 & 12.8 & 2.52 & 57 & 13.6 & 2.47 & -0.8 \\
& Masculino & 57 & 12.2 & 2.67 & 57 & 12.9 & 2.65 & -0.7 \\
& Total & $\mathbf{1 1 4}$ & $\mathbf{1 2 . 6}$ & $\mathbf{2 . 5 9}$ & $\mathbf{1 1 4}$ & $\mathbf{1 3 . 5}$ & $\mathbf{2 . 5 5}$ & $-\mathbf{0 . 9}$ \\
\hline \multirow{3}{*}{ Moorreees } & Femenino & 57 & 12.8 & 2.52 & 57 & 12.6 & 2.56 & 0.2 \\
& Masculino & 57 & 12.2 & 2.67 & 57 & 12.3 & 2.6 & -0.1 \\
& Total & $\mathbf{1 1 4}$ & $\mathbf{1 2 . 6}$ & $\mathbf{2 . 5 9}$ & $\mathbf{1 1 4}$ & $\mathbf{1 2 . 5}$ & $\mathbf{2 . 5 7}$ & $\mathbf{0 . 1}$ \\
\hline \multirow{3}{*}{ Nolla } & Femenino & 57 & 12.8 & 2.52 & 57 & 12.6 & 2.52 & 0.2 \\
& Masculino & 57 & 12.2 & 2.67 & 57 & 13 & 2.47 & -0.8 \\
& Total & $\mathbf{1 1 4}$ & $\mathbf{1 2 . 6}$ & $\mathbf{2 . 5 9}$ & $\mathbf{1 1 4}$ & $\mathbf{1 3}$ & $\mathbf{2 . 4 9}$ & $\mathbf{- 0 . 4}$ \\
\hline
\end{tabular}

\section{Gráfico 1}

\section{Comparación de los Errores de \\ Estimación por Método}

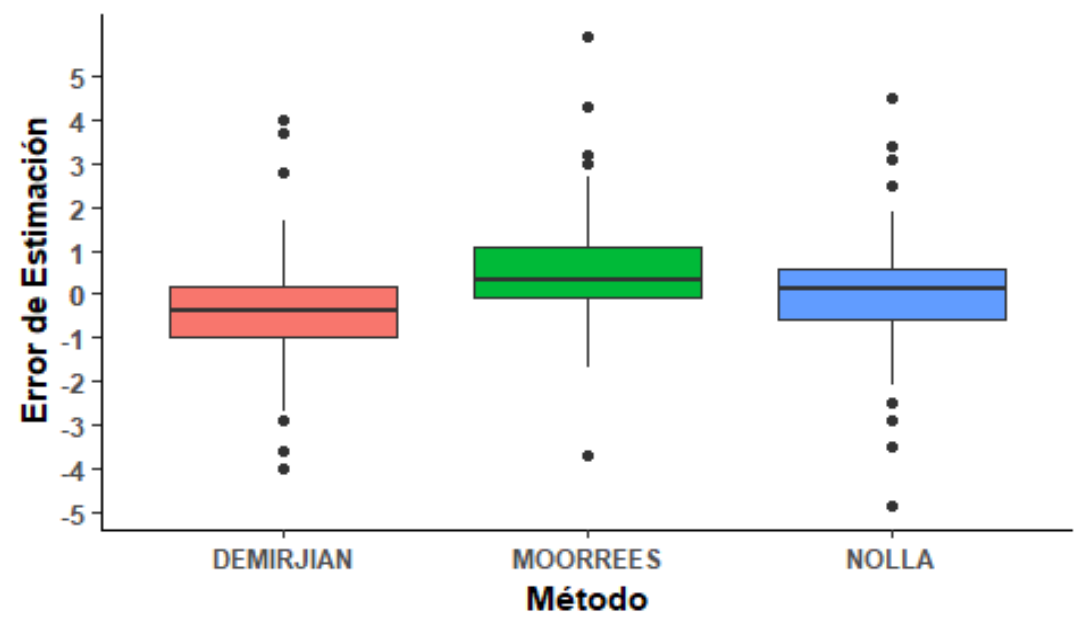

Metodo 自 DEMIRJIAN 它 MOORREes 自 NOLLA 
6 Revista de

Ciencias Forenses de Honduras

Cuadro 4: Error estimado por método y sexo

\begin{tabular}{clcccccc}
\hline Método & Genero & $\boldsymbol{n}$ & Media & Mediana & Sd & Min & Max \\
\hline \multirow{4}{*}{ Demirijian } & Femenino & 57 & -0.4 & -0.4 & 1.23 & -4 & 4 \\
& Masculino & 57 & -0.49 & -0.4 & 1.31 & -3.59 & 3.71 \\
& Total & $\mathbf{1 1 4}$ & $\mathbf{- 0 . 4 4}$ & $-\mathbf{0 . 4}$ & $\mathbf{1 . 2 7}$ & $\mathbf{- 4}$ & $\mathbf{4}$ \\
\hline \multirow{4}{*}{ Moorrees } & Femenino & 57 & 0.51 & 0.31 & 1.14 & -3.69 & 4.3 \\
& Masculino & 57 & 0.5 & 0.30 & 1.32 & -1.69 & 5.91 \\
& Total & $\mathbf{1 1 4}$ & $\mathbf{0 . 5}$ & $\mathbf{0 . 3 0}$ & $\mathbf{1 . 2 3}$ & $\mathbf{- 3 . 6 9}$ & $\mathbf{5 . 9 1}$ \\
\hline \multirow{3}{*}{ Nolla } & Femenino & 57 & 0.12 & 0.10 & 1.23 & -4.89 & 4.5 \\
& Masculino & 57 & -0.23 & -0.20 & 1.35 & -3.5 & 3.4 \\
& Total & $\mathbf{1 1 4}$ & $\mathbf{- 0 . 0 5}$ & $\mathbf{0 . 1 0}$ & $\mathbf{1 . 3}$ & $\mathbf{- 4 . 8 9}$ & $\mathbf{4 . 5}$ \\
\hline
\end{tabular}

\section{Gráfico $\mathbf{N}^{\circ} 2$}

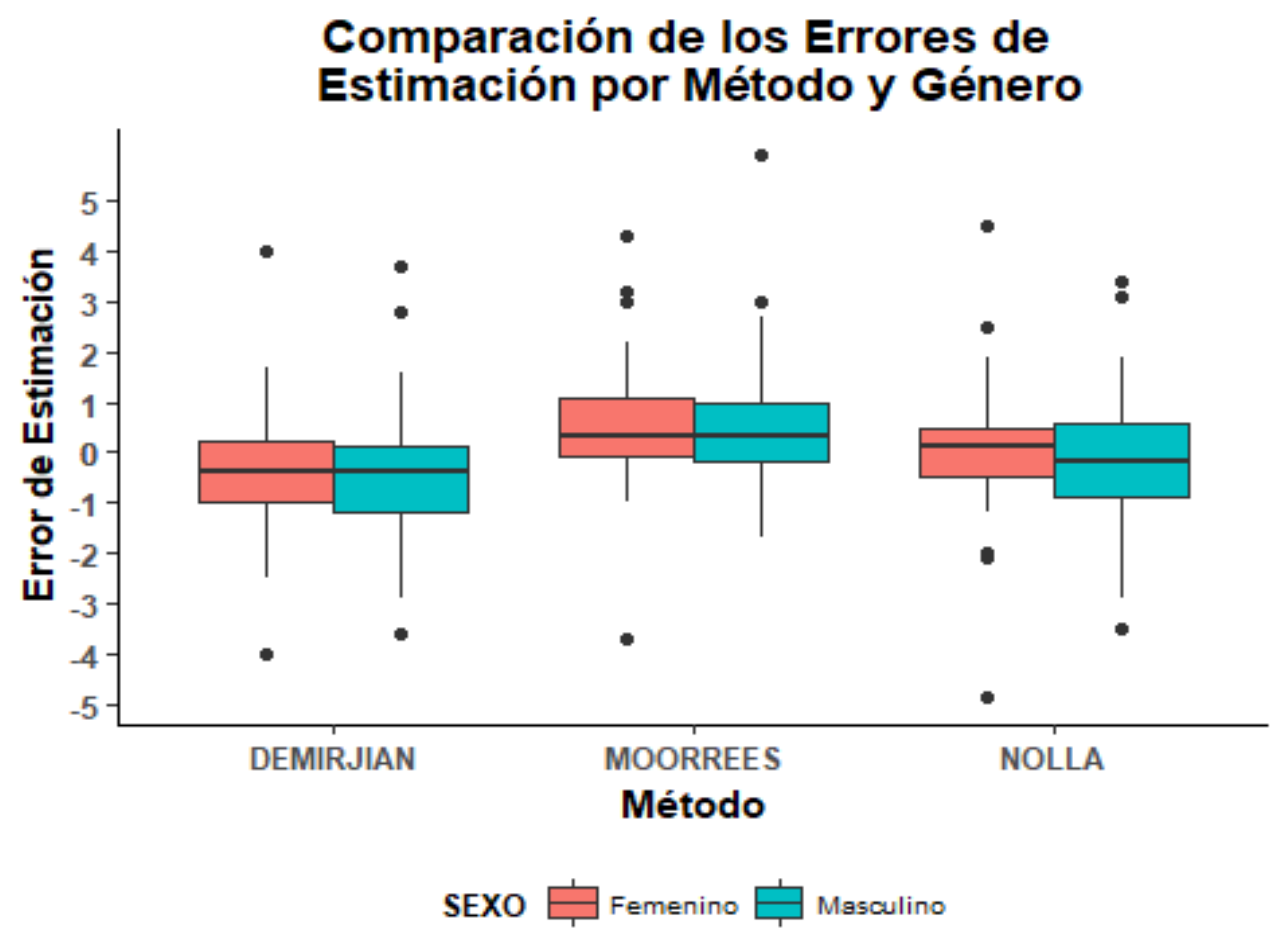

\section{DISCUSIÓN}

En la muestra analizada no se encontraron diferencias estadísticamente significativas entre la edad cronológica y la edad dental estimada indistintamente del método aplicado $(p=<0,05)$, lo que concuerda con lo reportado por Corral y col. en un estudio realizado en niños colombianos ${ }^{12}$; estudios realizados en Argentina ${ }^{13}$, Brasil ${ }^{14}$, Maracaibo, Venezuela ${ }^{15}$, muestran diferencias estadísticamente significativas entre la edad cronológica y la edad dental estimada por los métodos de Demirjian y Nolla. El error 
estimado total entre la edad dental estimada por método y la edad cronológica en la muestra analizada, no es significativo valor $p=<0,05$.

Nuestros resultados indican que Demirjian, subestima la edad indistintamente del sexo, Mooress y Nolla sobreestiman la edad en mujeres y la subestiman en hombres (Calculado por la diferencia de medianas entre la edad cronológica y la edad dental.) A nivel Latinoamericano son escasos los estudios realizados al respecto y no se encontraron estudios similares provenientes de poblaciones Centroamericanas, aunque estudios realizados en Venezuela y Brasil ${ }^{1,13-15}$ reportan una sobreestimación del método de Demirjian, sin embargo un estudio realizado en Colombia y otro en Venezuela por Cruz-Landeira y col., en un grupo de amerindios venezolanos reportaron subestimación con el método de Demirjian ${ }^{12,17}$, al igual que lo encontrado en nuestro estudio, lo que podría explicarse debido a factores nutricionales o al componente amerindio propio de la población hondureña. En general se ha descrito sobre y subestimación para este método, dependiendo de la población estudiada y el tamaño de la muestra.

Por otro lado, en nuestro estudio los métodos de Nolla y Mooress sobreestiman la edad en mujeres y la subestiman en hombres lo que podría estar asociado al proceso de maduración más tardío en los hombres que en las mujeres, aunque Wolf y Briceño-Marroquin encontraron que el sexo no influenciaba los cálculos de edad dental cuando se aplicó el método de Demirjian, en una muestra de niños alemanes $^{18}$. En un estudio realizado en niños venezolanos se encontró que el método de Nolla subestima la edad ${ }^{11}$, al igual que en un estudio realizado en el Reino Unido ${ }^{19}$.

Para la muestra analizada de niños hondureños, no se encontraron diferencias significativas entre la edad cronológica y la edad dental calculada, indistintamente del método utilizado, sin embargo, cuando se comparó el error estimado (EE) por método se observó que Nolla es más exacto que Moores y este a su vez más exacto que Demirjian. El método cuyo error estimado se acerca más a cero es el más exacto, Nolla $(E E=0.1)$, Moores $(E E=0.3)$, Demirjian $(E E=-0.4)$, para un valor $P=<0,05$.

En las ciencias forenses, es necesario que los métodos empleados proporcionen una edad estimada lo más cercana a la edad real ya que de ello depende el tratamiento civil o penal del individuo. Se recomienda para futuros estudios aumentar el tamaño de la muestra, estratificándola en grupos etarios.

\section{REFERENCIAS BIBLIOGRÁFICAS}

1.- Tineo F, Espina de Ferreira Al, Barrios F y col. Estimación de la edad cronológica con fines forenses, empleando la edad dental y la edad ósea en niños escolares en Maracaibo, estado Zulia. Acta odontol. Venez [Internet]. 2006 Ago. [citado 2018 Jun 27]; 44( 2 ): 184-191. Disponible en:

http://www.scielo.org.ve/scielo.php?script=sci a rttext\&pid=S0001-63652006000200006\&lng=es.

2.-Maldonado MB, Briem-Stamm AD, Métodos para estimación de edad dental: Un constante desafío para el odontólogo forense. Gac. int. cienc. forense.2013(6):12-22
3.-Pérez $M$, Herrera A, Moreno $S$, Moreno $F$. Estimación de la edad dental a través de seis métodos radiográficos en un grupo de afrodescendientes y mestizos caucasoides. Cuad. med. forense [Internet]. 2016 Dic [citado 2018 Jun 28]; 22( 3-4 ): 81-92. Disponible en:

http://scielo.isciii.es/scielo.php?script=sci arttex $\underline{\text { t\&pid=S1135-76062016000200004\&lng=es. }}$

4.-Demirjian A, Goldstein H, Tanner JM. A new system of dental age assessment. Ann Hum Biol. 1973; 45:211-27. 
5.- Demirjian A, Goldstein H. New systems for dental maturity based on seven and four teeth, Ann. Hum. Biol. (1976);3: 411-21.

6.- Willems G Et al. Dental age estimation in Belgian children: Demirjian's technique revisited, J. Forensic Sci. 46 (2001) 893-895

7.-Nolla CM. The development of the permanent teeth, J. Dent. Child. (1960); 27:254-266

8.- Moorrees CFA, Flanning EA, Hunt E. Age variation of formation stages for ten permanent teeth. J Dent Res. 1963; 42:1490-502.

9.- Bauer D. Constructing confidence sets using rank statistics. Journal of the American Statistical Association 1972: 67,687-690. Doi: $\underline{10.1080 / 01621459.1972 .10481279}$

10.- Royston P. An extension of Shapiro and Wilk's $\mathrm{W}$ test for normality to large samples. Applied Statistics, 1982:31, 176-180. Doi: $\underline{10.2307 / 2347986}$

11.- Medina AC, Blanco L. Accuracy of dental age estimation in Venezuelan children: comparison of Demirjian and Willems methods. Acta odontol. latinoam. [online]. 2014, vol.27, n.1 [citado 201808-27], pp.34-41. Disponible en: https://pdfs.semanticscholar.org/32dc/c5e96f03 006996115ada0cf07ae7aff72d64.pdf

12.-Corral C, García F, García J, León P, Herrera A.M, Martínez $C$, et al. Edad cronológica vs. edad dental en individuos de 5 a 19 años: un estudio comparativo con implicaciones forenses. Colomb Med., 41 (3) (2010), pp. 215-223
13.-Pobletto A, Giménez E. Edad dentaria: adecuación regional de los métodos de Nolla y Demirjian. UNcuyo. 2012; 6 (2): 37-42.

14.- Kurita L.M., Menezes A.V., Casanova M.S., Haiter-Neto F. Dental maturity as an indicator of chronological age: radiographic assessment of dental age in a Brazilian population. J Appl Oral Sci., 15 (2) (2007), pp. 99-104

15.-Martínez Gutiérrez VM, Ortega-Pertuz Al. Comparison of Nolla, Demirjian and Moorrees methods for dental age calculation for forensic purposes. Revista Odontológica Mexicana, Volume 21, Issue 3, July-September 2017, Pages e151-e159

16.- Ortega-Pertuz A, Martínez V. Estimación de la edad dental por el método de Demirjian y sus modificaciones en un grupo de venezolanos. Odous Científica. 2015; 16(1): 7-17 7

17.-Cruz-Landeira A, Linares-Argote J, MartínezRodríguez $M$, Rodríguez-Calvo $M S$, Otero $X L$, Concheiro L. Dental age estimation in Spanish and Venezuelan children. Comparison of Demirjian and Chaillet's scores. Int J Legal Med 2010; 124:105-112.

18.- Wolf et al. BMC Oral Health (2016) 16:120. DOI: $10.1186 / \mathrm{s} 12903-016-0315-8$

19.- Maber M, Liversidge HM, Hector MP. Accuracy of age estimation of radiographic methods using developing teeth. Forensic Sci Int. 2006 May 15;159 Suppl 1: S68-73. Epub 2006 Mar 14. DOI: $10.1016 /$ i.forsciint.2006.02.019 